\title{
The Cambrian Hopf Algebra
}

\author{
G. Chatel1" and V. Pilaud” \\ ${ }^{1}$ LIGM, Université Paris-Est Marne-la-Vallée, France \\ ${ }^{2}$ CNRS \& LIX, École Polytechnique, France
}

\begin{abstract}
Cambrian trees are oriented and labeled trees which fulfill local conditions around each node generalizing the conditions for classical binary search trees. Based on the bijective correspondence between signed permutations and leveled Cambrian trees, we define the Cambrian Hopf algebra generalizing J.-L. Loday and M. Ronco's algebra on binary trees. We describe combinatorially the products and coproducts of both the Cambrian algebra and its dual in terms of operations on Cambrian trees. Finally, we define multiplicative bases of the Cambrian algebra and study structural and combinatorial properties of their indecomposable elements.

Résumé. Les arbres Cambriens sont des arbres orientés et étiquetés qui satisfont des conditions locales autour de leurs noeuds généralisant les conditions des arbres binaires de recherche classiques. À partir de la correspondence bijective entre permutations signées et arbres Cambriens à niveau, nous définissons l'algèbre Cambrienne qui généralise l'algèbre sur les arbres binaires de J.-L. Loday et M. Ronco. Nous donnons une description combinatoire du produit et du coproduit aussi bien dans l'algèbre Cambrienne que dans sa duale en termes d'opérations sur les arbres Cambriens. Enfin, nous définissons des bases multiplicatives de l'algèbre Cambrienne et étudions les propriétés structurelles et énumératives de leurs éléments indécomposables.
\end{abstract}

Keywords: Combinatorial Hopf algebras, Cambrian lattices, Cambrian trees

The background of this paper is the fascinating interplay between the combinatorial, geometric and algebraic structures of permutations, binary trees and binary sequences:

$\star$ Combinatorially, the descent map from permutations to binary sequences factors via binary trees through the BST insertion and the canopy map. These maps define lattice homomorphisms from the weak order via the Tamari lattice to the boolean lattice.

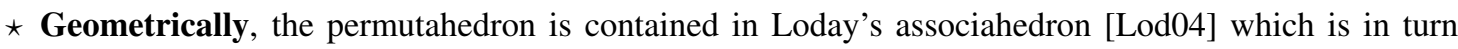
contained in the parallelepiped generated by the simple roots. See Figure 1 .

* Algebraically, these maps translate to Hopf algebra inclusions from M. Malvenuto and C. Reutenauer's algebra on permutations [MR95] via J.-L. Loday and M. Ronco's algebra on binary trees [LR98] to L. Solomon's descent algebra [Sol76].

These structures and their connections have been partially extended in several directions, see e.g. [CD06, Pos09, BPR12, NT14]. This paper explores combinatorial and algebraic aspects of Hopf algebras related

*gregory. chateleuniv-paris-est.fr

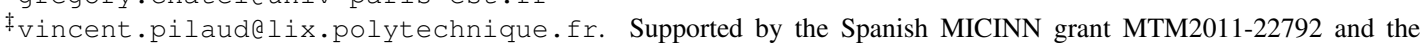
French ANR grant EGOS (12 JS02 002 01).

1365-8050 @ 2015 Discrete Mathematics and Theoretical Computer Science (DMTCS), Nancy, France 


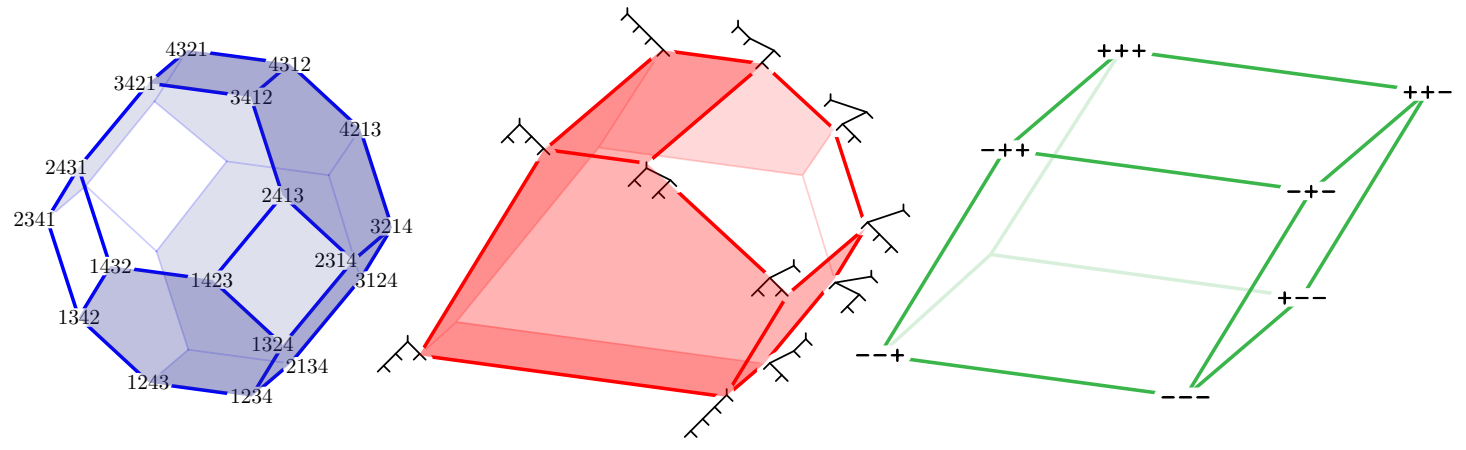

Fig. 1: The 3-dimensional permutahedron (blue, left), Loday's associahedron (red, middle), and parallelepiped (green, right). Shaded facets are preserved to get the next polytope.

to N. Reading's type $A$ Cambrian lattices [Rea06] and their polytopal realizations by C. Hohlweg and C. Lange [HL07]. N. Reading provides in [Rea06] a procedure to map a signed permutation of $\mathfrak{S}_{n}$ into a triangulation of a certain convex $(n+3)$-gon. The dual trees of these triangulations naturally extend rooted binary trees and were introduced and studied as "spines" [LP13] or "mixed cobinary trees" [IO13]. We prefer here the term "Cambrian trees" in reference to N. Reading's work. The map $\kappa$ from signed permutations to Cambrian trees is known to encode combinatorial and geometric properties of the Cambrian structures: the Cambrian lattice is the quotient of the weak order under the fibers of $\kappa$, each maximal cone of the Cambrian fan is the incidence cone of a Cambrian tree $\mathrm{T}$ and is refined by the braid cones of the permutations in the fiber $\kappa^{-1}(\mathrm{~T})$, etc.

In this paper, we use this map $\kappa$ for algebraic purposes. We introduce the Cambrian Hopf algebra Camb as a subalgebra of the Hopf algebra $\mathrm{FQSym}_{ \pm}$on signed permutations, and the dual Cambrian algebra Camb* as a quotient algebra of the dual Hopf algebra FQSym ${ }_{ \pm}^{*}$. Their bases are indexed by all Cambrian trees. Our approach extends that of F. Hivert, J.-C. Novelli and J.-Y. Thibon [HNT05] to construct J.-L. Loday and M. Ronco's Hopf algebra on binary trees [LR98] as a subalgebra of C. Malvenuto and C. Reutenauer's Hopf algebra on permutations [MR95]. We also use this map $\kappa$ to describe both the product and coproduct in the algebras Camb and Camb* in terms of simple combinatorial operations on Cambrian trees. From the combinatorial description of the product in Camb, we derive multiplicative bases of the Cambrian algebra Camb and study the structural and enumerative properties of their indecomposable elements. We refer to [CP14] for detailed proofs and further aspects of the Cambrian algebra.

\section{Cambrian trees}

\subsection{Cambrian trees and increasing trees}

Consider a directed tree $\mathrm{T}$ on a vertex set $\mathrm{V}$ and a vertex $v \in \mathrm{V}$. We call children (resp. parents) of $v$ the sources of the incoming arcs (resp. the targets of the outgoing arcs) at $v$ and descendants (resp. ancestor) subtrees of $v$ the subtrees attached to them. The main characters of our paper are the following trees, which generalize standard binary search trees. Our definition is adapted from [1013, LP13].

Definition 1 A Cambrian tree is a directed tree $\mathrm{T}$ with vertex set $\mathrm{V}$ endowed with a bijective vertex labeling $p: \mathrm{V} \rightarrow[n]$ such that for each vertex $v \in \mathrm{V}$, 

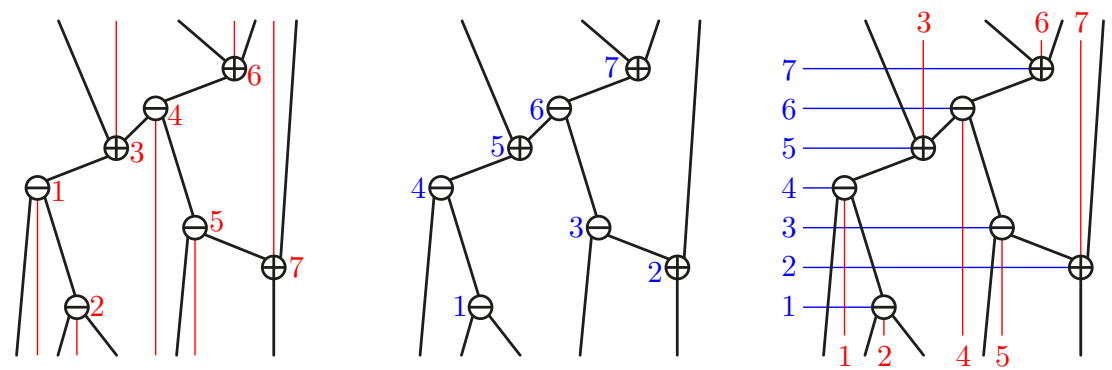

Fig. 2: A Cambrian tree (left), an increasing tree (middle), and a leveled Cambrian tree (right).

(i) $v$ has either one parent and two children (its descendant subtrees are called left and right subtrees) or one child and two parents (its ancestor subtrees are called left and right subtrees);

(ii) all labels are smaller (resp. larger) than $p(v)$ in the left (resp. right) subtree of $v$.

The signature of $\mathrm{T}$ is the $n$-tuple $\varepsilon(\mathrm{T}) \in \pm^{n}$ defined by $\varepsilon(\mathrm{T})_{p(v)}=-$ if $v$ has two children and + if $v$ has two parents. Denote by $\operatorname{Camb}(\varepsilon)$ the set of Cambrian trees with signature $\varepsilon$, by $\operatorname{Camb}(n)$ the set of all Cambrian trees on $n$ vertices, and by Camb the set of all Cambrian trees.

Definition 2 An increasing tree is a directed tree $\mathrm{T}$ with vertex set $\mathrm{V}$ endowed with a bijective vertex labeling $q: \mathrm{V} \rightarrow[n]$ such that $v \rightarrow w$ in $\mathrm{T}$ implies $q(v)<q(w)$.

Definition 3 A leveled Cambrian tree is a directed tree $\mathrm{T}$ with vertex set $\mathrm{V}$ endowed with two bijective vertex labelings $p, q: V \rightarrow[n]$ which respectively define a Cambrian and an increasing tree.

In other words, a leveled Cambrian tree is a Cambrian tree endowed with a linear extension of its transitive closure. Figure 2 provides examples of a Cambrian tree (left), an increasing tree (middle), and a leveled Cambrian tree (right). All edges are oriented bottom-up. Throughout the paper, we represent leveled Cambrian trees on an $(n \times n)$-grid as follows (see Figure 2):

(i) each vertex $v$ appears at position $(p(v), q(v))$;

(ii) negative vertices (with one parent and two children) are represented by $\ominus$, while positive vertices (with one child and two parents) are represented by $\oplus$;

(iii) we sometimes draw a vertical red wall below the negative vertices and above the positive vertices to mark the separation between the left and right subtrees of each vertex.

Proposition 4 ([LP13, IO13|) For any signature $\varepsilon \in \pm^{n}$, the number of $\varepsilon$-Cambrian trees is the Catalan number $C_{n}=\frac{1}{n+1}\left(\begin{array}{c}2 n \\ n\end{array}\right)$. Therefore, $|\operatorname{Camb}(n)|=2^{n} C_{n}$.

\subsection{Cambrian correspondence}

We represent graphically a permutation $\tau \in \mathfrak{S}_{n}$ by the $(n \times n)$-table, with rows labeled by positions from bottom to top and columns labeled by values from left to right, and with a dot in row $i$ and column $\tau(i)$ for all $i \in[n]$. (This unusual choice of orientation is necessary to fit later with the existing constructions of [LR98, HNT05].)

A signed permutation is a permutation table where each dot receives a + or - sign, see the top left corner of Figure 3 . We could equivalently think of a permutation where the positions or the values receive 


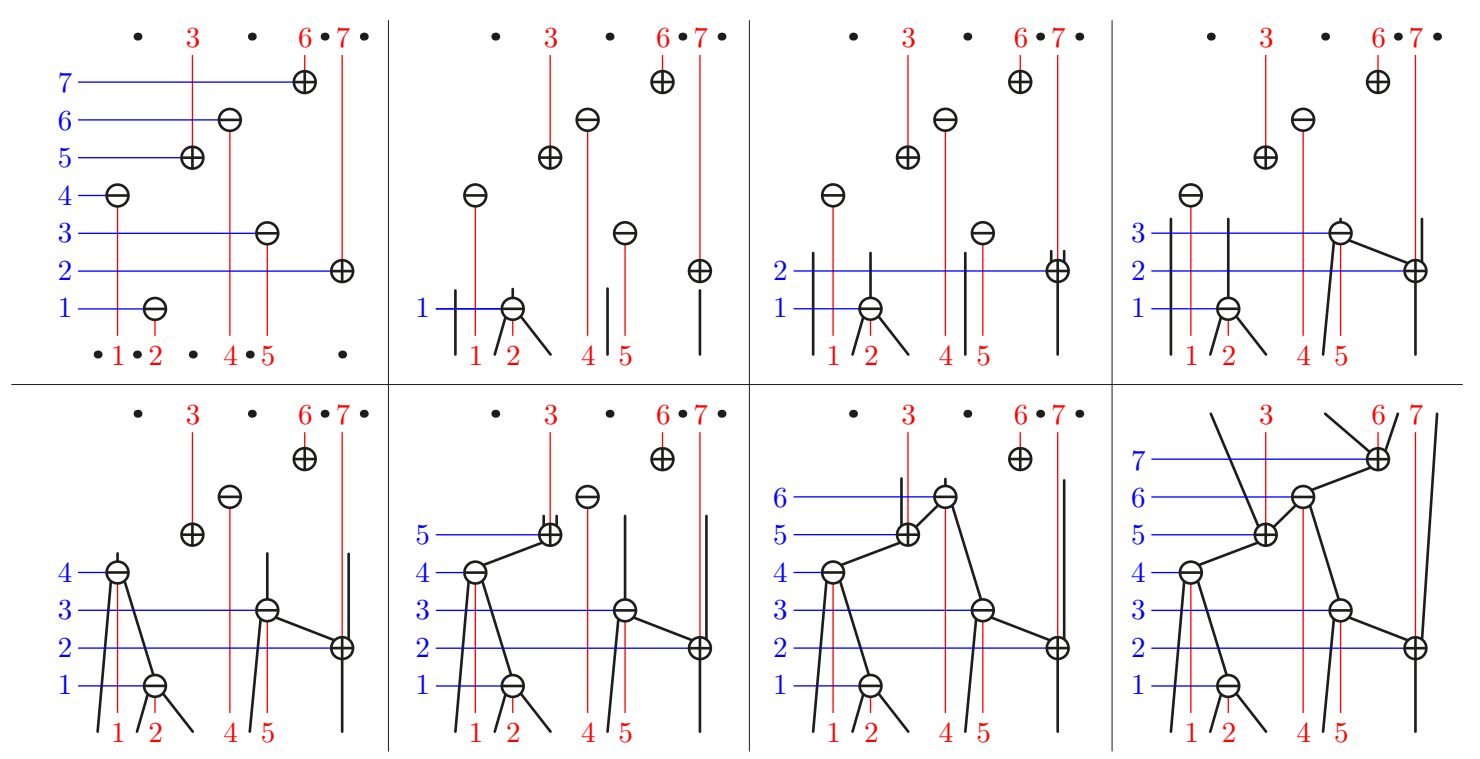

Fig. 3: The insertion algorithm on the signed permutation $\underline{2} \overline{7} \underline{51} \overline{3} \underline{4} \overline{6}$.

a sign, but it will be useful later to switch the signature from positions to values. The $p$-signature (resp. $v$ signature) of a signed permutation $\tau$ is the sequence $\varepsilon_{p}(\tau)$ (resp. $\varepsilon_{v}(\tau)$ ) of signs of $\tau$ ordered by positions from bottom to top (resp. by values from left to right). For $\varepsilon \in \pm^{n}$, we denote by $\mathfrak{S}_{\varepsilon}$ (resp. by $\mathfrak{S}^{\varepsilon}$ ) the set of signed permutations $\tau$ with p-signature $\varepsilon_{p}(\tau)=\varepsilon$ (resp. with v-signature $\varepsilon_{v}(\tau)=\varepsilon$ ). Finally, we denote the set of all signed permutations by $\mathfrak{S}_{ \pm}:=\bigsqcup_{\varepsilon \in \pm^{*}} \mathfrak{S}_{\varepsilon}=\bigsqcup_{\varepsilon \in \pm^{*}} \mathfrak{S}^{\varepsilon}$.

In concrete examples, we underline negative positions/values and overline positive positions/values: for example, we write $2 \overline{7} \underline{51} \overline{3} \underline{\underline{6}}$ for the signed permutation represented on the top left corner of Figure 3 . where $\tau=[2,7,5,1,3,4,6], \varepsilon_{p}=-+--+-+$ and $\varepsilon_{v}=--+--++$.

Following [LP13], we now present an algorithm to construct a leveled $\varepsilon$-Cambrian tree $\Theta(\tau)$ from a signed permutation $\tau \in \mathfrak{S}^{\varepsilon}$. Figure 3 illustrates this algorithm on the permutation $2 \overline{7} \underline{51} \overline{3} \underline{\underline{6}}$. As a preprocessing, we represent the table of $\tau$ (with signed dots in positions $(\tau(i), i)$ for $i \in[n]$ ) and draw a vertical wall below the negative vertices and above the positive vertices. We then sweep the table from bottom to top (thus reading the permutation $\tau$ from left to right) as follows. The procedure starts with an incoming strand in between any two consecutive negative values. A negative dot $\ominus$ connects the two strands immediately to its left and immediately to its right to form a unique outgoing strand. A positive $\operatorname{dot} \oplus$ separates the only visible strand (not hidden by a wall) into two outgoing strands. The procedure finishes with an outgoing strand in between any two consecutive positive values. See Figure 3

Proposition 5 ([LP13]) The map $\Theta$ is a bijection from signed permutations to leveled Cambrian trees.

Definition 6 Given a signed permutation $\tau \in \mathfrak{S}^{\varepsilon}$, its $\mathbf{P}$-symbol is the insertion Cambrian tree $\mathbf{P}(\tau)$ defined by $\Theta(\tau)$ and its $\mathbf{Q}$-symbol is the recording increasing tree $\mathbf{Q}(\tau)$ defined by $\Theta(\tau)$. 


\subsection{Cambrian congruence}

Similarly to the sylvester congruence in [HNT05], we now characterize by a congruence the signed permutations $\tau \in \mathfrak{S}^{\varepsilon}$ with the same $\mathbf{P}$-symbol $\mathbf{P}(\tau)$. The definition appears in N. Reading's work [Rea06].

Definition 7 ([|Rea06]) For a signature $\varepsilon \in \pm^{n}$, the $\varepsilon$-Cambrian congruence is the equivalence relation on $\mathfrak{S}^{\varepsilon}$ defined as the transitive closure of the rewriting rules

$$
U a c V b W \equiv{ }_{\varepsilon} U c a V b W \quad \text { if } \varepsilon_{b}=-\quad \text { and } \quad U b V a c W \equiv{ }_{\varepsilon} U b V c a W \quad \text { if } \varepsilon_{b}=+
$$

where $a<b<c \in[n]$ and $U, V, W \in[n]^{*}$. The Cambrian congruence is the congruence $\equiv:=\bigsqcup_{\varepsilon \in \pm^{*}} \equiv_{\varepsilon}$ on all signed permutations $\mathfrak{S}_{ \pm}$obtained as the union of all $\varepsilon$-Cambrian congruences.

Proposition 8 Two signed permutations $\tau, \tau^{\prime} \in \mathfrak{S}^{\varepsilon}$ are $\varepsilon$-Cambrian congruent if and only if they have the same $\mathbf{P}$-symbol: $\tau \equiv_{\varepsilon} \tau^{\prime} \Longleftrightarrow \mathbf{P}(\tau)=\mathbf{P}\left(\tau^{\prime}\right)$.

Proposition 9 The signed permutations $\tau \in \mathfrak{S}^{\varepsilon}$ such that $\mathbf{P}(\tau)=\mathrm{T}$ are precisely the linear extensions of (the transitive closure of) $\mathrm{T}$.

Remember that the (right) weak order on $\mathfrak{S}^{\varepsilon}$ is defined as the inclusion order of coinversions, where a coinversion of $\tau \in \mathfrak{S}^{\varepsilon}$ is a pair of values $i<j$ such that $\tau^{-1}(i)>\tau^{-1}(j)$ (no matter the signs on $\tau$ ). The following statement is due to N. Reading [Rea06].

Proposition 10 ([[Rea06]) All $\varepsilon$-Cambrian classes are intervals of the weak order on $\mathfrak{S}^{\varepsilon}$.

\subsection{Rotations and Cambrian lattices}

Definition 11 Let $i \rightarrow j$ be an edge in a Cambrian tree $\mathrm{T}$, with $i<j$. Let L denote the left subtree of $i$ and $B$ denote the remaining incoming subtree of $i$, and similarly, let $R$ denote the right subtree of $j$ and $A$ denote the remaining outgoing subtree of $j$. Let $\mathrm{T}^{\prime}$ be the oriented tree obtained from $\mathrm{T}$ just reversing the orientation of $i \rightarrow j$ and attaching the subtrees $L$ and $A$ to $i$ and the subtrees $B$ and $R$ to $j$. The transformation from $\mathrm{T}$ to $\mathrm{T}^{\prime}$ is called rotation of the edge $i \rightarrow j$. See Figure 4
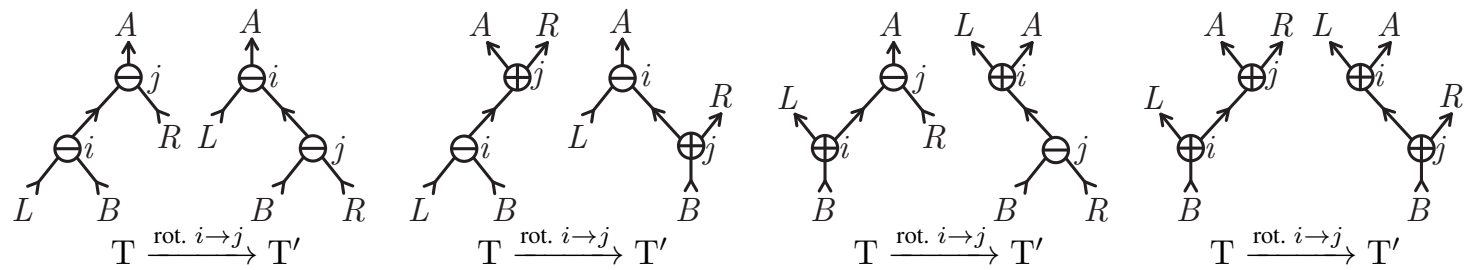

Fig. 4: Rotations in Cambrian trees: the tree $\mathrm{T}$ (top) is transformed into the tree $\mathrm{T}^{\prime}$ (bottom) by rotation of the edge $i \rightarrow j$. The four cases correspond to the possible signs of $i$ and $j$.

An edge cut in a Cambrian tree $\mathrm{T}$ is the ordered partition $(X \| Y)$ of the vertices of $\mathrm{T}$ into the set $X$ of vertices in the source set and the set $Y$ of vertices in the target set of an oriented edge of $\mathrm{T}$.

Proposition 12 ([LP13]) The result $\mathrm{T}^{\prime}$ of the rotation of an edge $i \rightarrow j$ in a $\varepsilon$-Cambrian tree $\mathrm{T}$ is a $\varepsilon$-Cambrian tree. Moreover, $\mathrm{T}^{\prime}$ is the unique $\varepsilon$-Cambrian tree with the same edge cuts as $\mathrm{T}$, except the cut defined by the edge $i \rightarrow j$. 

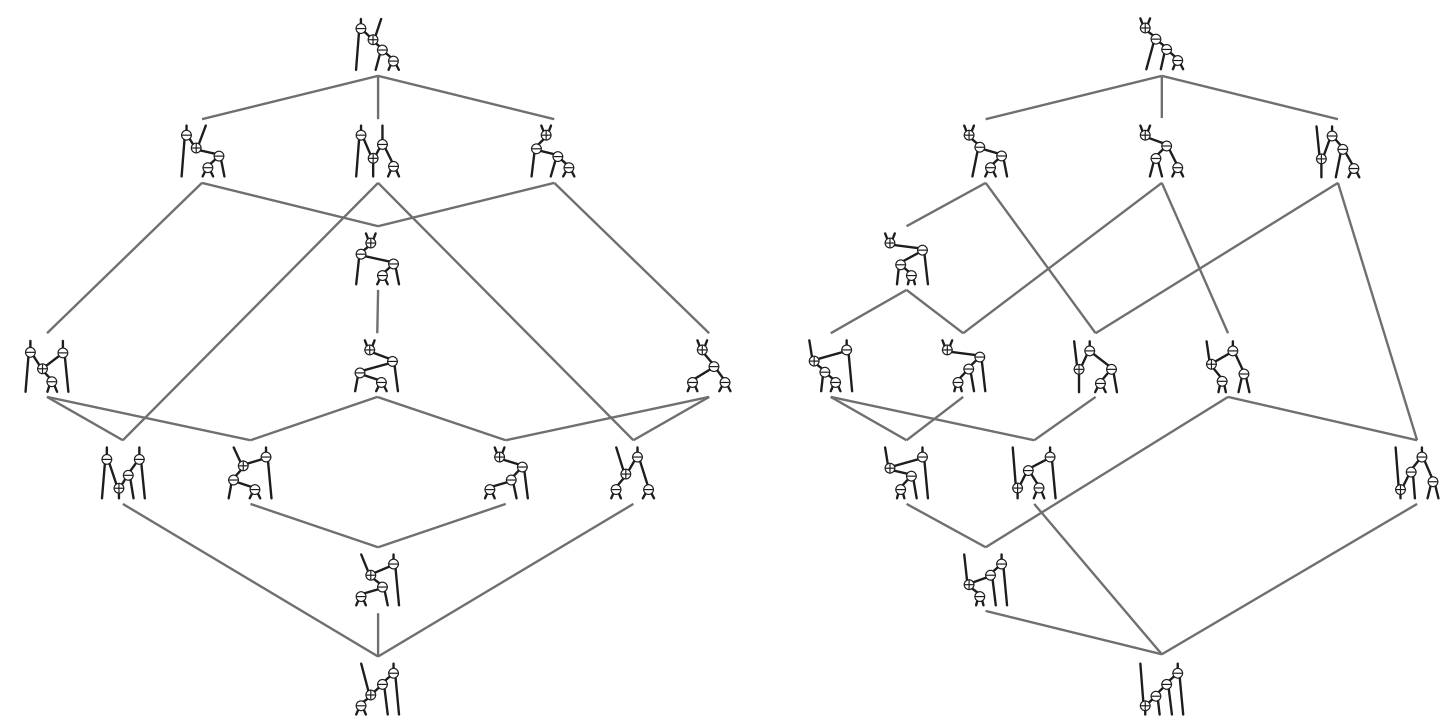

Fig. 5: The $\varepsilon$-Cambrian lattices on $\varepsilon$-Cambrian trees, for the signatures $\varepsilon=-+--$ (left) and $\varepsilon=+---$ (right).

Define the increasing rotation graph on $\operatorname{Camb}(\varepsilon)$ to be the graph whose vertices are the $\varepsilon$-Cambrian trees and whose arcs are increasing rotations $\mathrm{T} \rightarrow \mathrm{T}^{\prime}$, i.e. where the edge $i \rightarrow j$ in $\mathrm{T}$ is reversed to the edge $i \leftarrow j$ in $\mathrm{T}^{\prime}$ for $i<j$. See Figure 5 for an illustration.

Proposition 13 ([Rea06]) The transitive closure of the increasing rotation graph on $\mathrm{Camb}(\varepsilon)$ is a lattice, called $\varepsilon$-Cambrian lattice. The map $\mathbf{P}: \mathfrak{S}^{\varepsilon} \rightarrow \operatorname{Camb}(\varepsilon)$ defines a lattice homomorphism from the weak order on $\mathfrak{S}^{\varepsilon}$ to the $\varepsilon$-Cambrian lattice on $\operatorname{Camb}(\varepsilon)$.

\section{Cambrian Hopf Algebra}

\subsection{Signed shuffle and convolution}

For $n, n^{\prime} \in \mathbb{N}$, let $\mathfrak{S}^{\left(n, n^{\prime}\right)}:=\left\{\tau \in \mathfrak{S}_{n+n^{\prime}} \mid \tau(1)<\cdots<\tau(n)\right.$ and $\left.\tau(n+1)<\cdots<\tau\left(n+n^{\prime}\right)\right\}$ denote the set of permutations of $\mathfrak{S}_{n+n^{\prime}}$ with at most one descent, at position $n$. The shifted concatenation $\tau \bar{\tau}^{\prime}$, the shifted shuffle product $\tau \bar{\amalg} \tau^{\prime}$, and the convolution $\tau \star \tau^{\prime}$ of two permutations $\tau \in \mathfrak{S}_{n}$ and $\tau^{\prime} \in \mathfrak{S}_{n^{\prime}}$ are classically defined by

$$
\begin{gathered}
\tau \bar{\tau}^{\prime}:=\left[\tau(1), \ldots, \tau(n), \tau^{\prime}(1)+n, \ldots, \tau^{\prime}\left(n^{\prime}\right)+n\right] \in \mathfrak{S}_{n+n^{\prime}}, \\
\tau \bar{\Psi} \tau^{\prime}:=\left\{\left(\tau \bar{\tau}^{\prime}\right) \circ \pi^{-1} \mid \pi \in \mathfrak{S}^{\left(n, n^{\prime}\right)}\right\} \quad \text { and } \quad \tau \star \tau^{\prime}:=\left\{\pi \circ\left(\tau \bar{\tau}^{\prime}\right) \mid \pi \in \mathfrak{S}^{\left(n, n^{\prime}\right)}\right\} .
\end{gathered}
$$

These definitions extend to signed permutations. The signed shifted shuffle product $\tau \bar{\Psi} \tau^{\prime}$ is the shifted product of the permutations where signs travel with their values, while the signed convolution $\tau \star \tau^{\prime}$ is the convolution of the permutations where signs stay at their positions. For example,

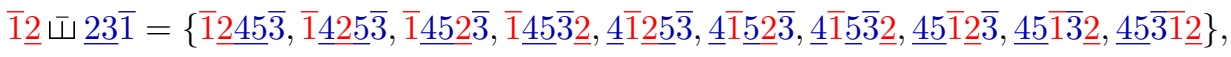

$$
\begin{aligned}
& \overline{1} \underline{2} \star \underline{23} \overline{1}=\{\overline{1} \underline{245} \overline{3}, \overline{1} \underline{345} \overline{2}, \overline{1} \underline{435} \overline{2}, \overline{1} \underline{534} \overline{2}, \overline{2} \underline{345} \overline{1}, \overline{2} \underline{435} \overline{1}, \overline{2} \underline{54} \overline{1}, \overline{3} \underline{425} \overline{1}, \overline{3} \underline{524} \overline{1}, \overline{4} \underline{523} \overline{1}\} .
\end{aligned}
$$




\subsection{Subalgebra of $\mathrm{FQSym}_{ \pm}$}

We denote by FQSym $m_{ \pm}$the Hopf algebra with basis $\left(\mathbb{F}_{\tau}\right)_{\tau \in \mathfrak{S}_{ \pm}}$and whose product and coproduct are defined by

$$
\mathbb{F}_{\tau} \cdot \mathbb{F}_{\tau^{\prime}}=\sum_{\sigma \in \tau \varpi \tau^{\prime}} \mathbb{F}_{\sigma} \quad \text { and } \quad \Delta \mathbb{F}_{\sigma}=\sum_{\sigma \in \tau \star \tau^{\prime}} \mathbb{F}_{\tau} \otimes \mathbb{F}_{\tau^{\prime}}
$$

It naturally extends to signed permutations the Hopf algebra FQSym on permutations defined by C. Malvenuto and C. Reutenauer [MR95].

We denote by Camb the vector subspace of $\mathrm{FQSym}_{ \pm}$generated by the elements

$$
\mathbb{P}_{\mathrm{T}}:=\sum_{\substack{\tau \in \mathfrak{S}_{ \pm} \\ \mathbf{P}(\tau)=\mathrm{T}}} \mathbb{F}_{\tau}=\sum_{\tau \in \mathcal{L}(\mathrm{T})} \mathbb{F}_{\tau}
$$

for all Cambrian trees T. For example, for the Cambrian tree of Figure 2](left), we have

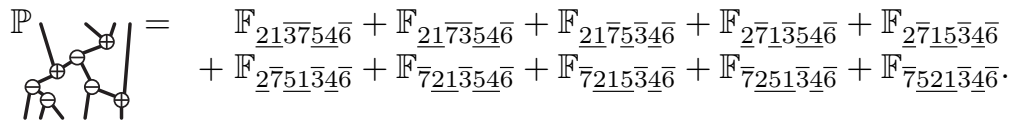

Theorem 14 Camb is a Hopf subalgebra of $\mathrm{FQSym}_{ \pm}$.

In the remaining of this section, we describe combinatorially the product and coproduct of $\mathbb{P}$-basis elements of Camb in terms of operations on Cambrian trees.

PRODUCT The product in the Cambrian algebra can be described in terms of intervals in Cambrian lattices. Given two Cambrian trees $T, T^{\prime}$, we denote by $T \backslash \bar{T}^{\prime}$ the tree obtained by grafting the rightmost outgoing leaf of $\mathrm{T}$ on the leftmost incoming leaf of $\mathrm{T}$ and shifting all labels of $\mathrm{T}^{\prime}$. Note that the resulting tree is $\varepsilon \varepsilon^{\prime}$-Cambrian, where $\varepsilon \varepsilon^{\prime}$ is the concatenation of the signatures $\varepsilon=\varepsilon(\mathrm{T})$ and $\varepsilon^{\prime}=\varepsilon\left(\mathrm{T}^{\prime}\right)$. We define similarly $\mathrm{T} / \overline{\mathrm{T}}^{\prime}$. An example is given in Figure 6 (left).

Proposition 15 For any Cambrian trees $\mathrm{T}, \mathrm{T}^{\prime}$, we have $\mathbb{P}_{\mathrm{T}} \cdot \mathbb{P}_{\mathrm{T}^{\prime}}=\sum_{\mathrm{S}} \mathbb{P}_{\mathrm{S}}$, where $\mathrm{S}$ runs over the interval between $\mathrm{T} \backslash \overline{\mathrm{T}}^{\prime}$ and $\mathrm{T} / \overline{\mathrm{T}}^{\prime}$ in the $\varepsilon(\mathrm{T}) \varepsilon\left(\mathrm{T}^{\prime}\right)$-Cambrian lattice.

For example, we can compute the product

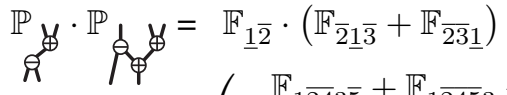

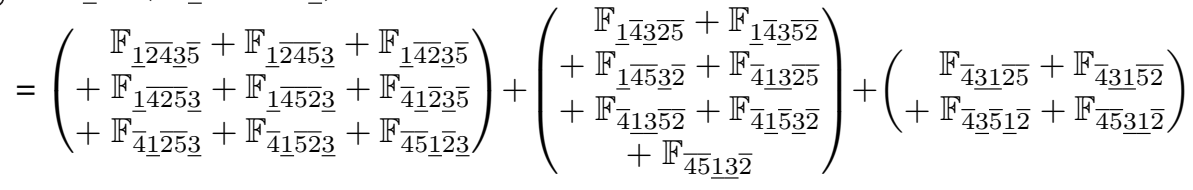

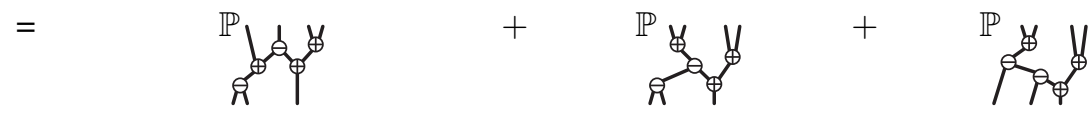

The first equality is obtained by computing the linear extensions of the two factors, the second by computing the shuffle product and grouping terms according to their $\mathbf{P}$-symbol, displayed in the last line. Proposition 15 enables us to shortcut the computation by avoiding to resort to the $\mathbb{F}$-basis. 

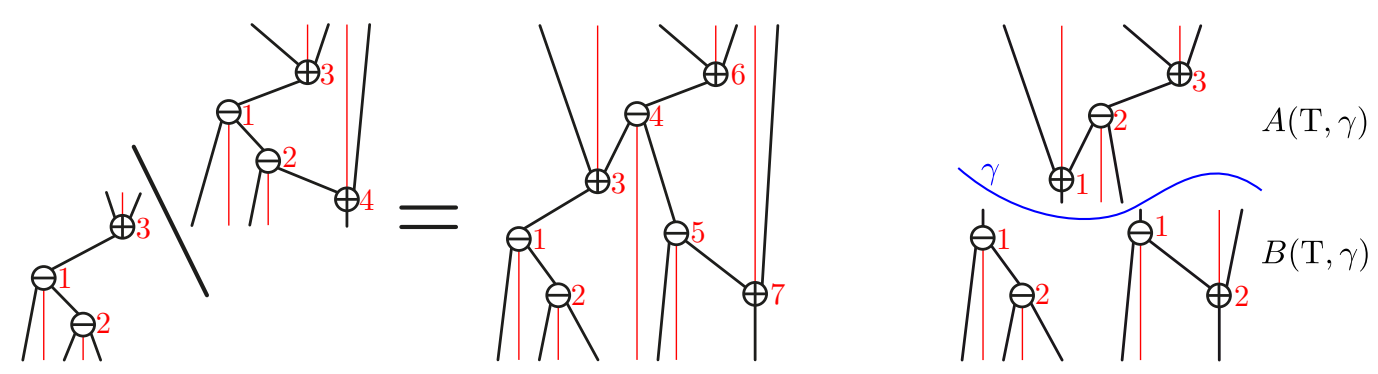

Fig. 6: (Left) The tree $T \backslash \overline{\mathrm{T}}^{\prime}$ obtained by identifying the rightmost outgoing leaf of $\mathrm{T}$ with the leftmost incoming leaf of T. (Right) A cut $\gamma$ of a Cambrian tree $\mathrm{T}$ defines two forests $A(\mathrm{~T}, \gamma)$ and $B(\mathrm{~T}, \gamma)$.

COPRODUCT The coproduct in the Cambrian algebra can also be described in combinatorial terms. Define a cut of a Cambrian tree $\mathrm{S}$ to be a set $\gamma$ of edges such that any geodesic vertical path in $\mathrm{S}$ from a down leaf to an up leaf contains precisely one edge of $\gamma$. Such a cut separates the tree T into two forests, one above $\gamma$ and one below $\gamma$, denoted $A(\mathrm{~S}, \gamma)$ and $B(\mathrm{~S}, \gamma)$, respectively. An example is given in Figure6(right).

Proposition 16 For any Cambrian tree $\mathrm{S}$, we have $\triangle \mathbb{P}_{\mathrm{S}}=\sum_{\gamma}\left(\prod_{\mathrm{T} \in B(\mathrm{~S}, \gamma)} \mathbb{P}_{\mathrm{T}}\right) \otimes\left(\prod_{\mathrm{T}^{\prime} \in A(\mathrm{~S}, \gamma)} \mathbb{P}_{\mathrm{T}^{\prime}}\right)$, where $\gamma$ runs over all cuts of $\mathrm{S}$.

For example, we can compute the coproduct

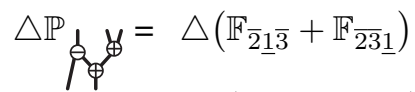

$$
\begin{aligned}
& =1 \otimes\left(\mathbb{F}_{\overline{2} \underline{1} \overline{3}}+\mathbb{F}_{\overline{23} \underline{1}}\right)+\mathbb{F}_{\overline{1}} \otimes \mathbb{F}_{\underline{1} \overline{2}}+\mathbb{F}_{\overline{1}} \otimes \mathbb{F}_{\overline{2} \underline{1}}+\mathbb{F}_{\overline{2} \underline{1}} \otimes \mathbb{F}_{\overline{1}}+\mathbb{F}_{\overline{12}} \otimes \mathbb{F}_{\underline{1}}+\left(\mathbb{F}_{\overline{2}} \underline{\overline{3}}+\mathbb{F}_{\overline{23} \underline{1}}\right) \otimes 1
\end{aligned}
$$

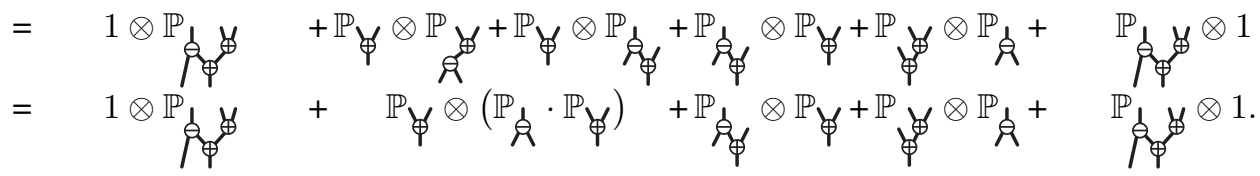

Proposition 16 enables us to shortcut the computation by avoiding to resort to the $\mathbb{F}$-basis. We compute directly the last line, which corresponds to the five possible cuts of the Cambrian tree $p_{p}$.

Remark 17 A different generalization of J.-L. Loday and M. Ronco's algebra was studied by N. Reading in [Rea05]. His idea was to construct a subalgebra of C. Malvenuto and C. Reutenauer's algebra FQSym using equivalent classes of a congruence relation defined as the union $\bigcup_{n \in \mathbb{N}} \equiv_{\varepsilon_{n}}$ of $\varepsilon_{n}$-Cambrian relation for one fixed signature $\varepsilon_{n} \in \pm^{n}$ for each $n \in \mathbb{N}$. In order to obtain a valid Hopf algebra, the choice of $\left(\varepsilon_{n}\right)_{n \in \mathbb{N}}$ has to satisfy certain compatibility relations: N. Reading characterizes the "translational" (resp. "insertional") families $\equiv_{n}$ of lattice congruences on $\mathfrak{S}_{n}$ for which the sums over the elements of the congruence classes of $\left(\equiv_{n}\right)_{n \in \mathbb{N}}$ form the basis of a subalgebra (resp. subcoalgebra) of FQSym. These conditions make the choice of $\left(\varepsilon_{n}\right)_{n \in \mathbb{N}}$ rather constrained. In contrast, by constructing a subalgebra of $\mathrm{FQSym}_{ \pm}$rather than $\mathrm{FQSym}$, we consider simultaneously all Cambrian relations for all signatures. In particular, our Cambrian algebra contains all Hopf algebras of [Rea05] as subalgebras. 


\subsection{Quotient algebra of FQSym ${ }_{ \pm}^{*}$}

We switch to the dual Hopf algebra FQSym $m_{ \pm}^{*}$ with basis $\left(\mathbb{G}_{\tau}\right)_{\tau \in \mathfrak{S}_{ \pm}}$and whose product and coproduct are defined by

$$
\mathbb{G}_{\tau} \cdot \mathbb{G}_{\tau^{\prime}}=\sum_{\sigma \in \tau \star \tau^{\prime}} \mathbb{G}_{\sigma} \quad \text { and } \quad \triangle \mathbb{G}_{\sigma}=\sum_{\sigma \in \tau \amalg \tau^{\prime}} \mathbb{G}_{\tau} \otimes \mathbb{G}_{\tau^{\prime}} .
$$

The following statement is automatic from Theorem 14

Theorem 18 The graded dual Camb* of the Cambrian algebra is isomorphic to the image of $\mathrm{FQSym}_{ \pm}^{*}$ under the canonical projection $\pi: \mathbb{C}\langle A\rangle \longrightarrow \mathbb{C}\langle A\rangle / \equiv$, where $\equiv$ denotes the Cambrian congruence. The dual basis $\mathbb{Q}_{\mathrm{T}}$ of $\mathbb{P}_{\mathrm{T}}$ is expressed as $\mathbb{Q}_{\mathrm{T}}=\pi\left(\mathbb{G}_{\tau}\right)$, where $\tau$ is any linear extension of $\mathrm{T}$.

Similarly as in the previous section, we can describe combinatorially the product and coproduct of $\mathbb{Q}$-basis elements of Camb* in terms of operations on Cambrian trees.

PRODUCT Call gaps the $n+1$ positions between two consecutive integers of $[n]$, including the position before 1 and the position after $n$. A gap $\gamma$ defines a geodesic vertical path $\lambda(\mathrm{T}, \gamma)$ in a Cambrian tree $\mathrm{T}$ from the bottom leaf which lies in the same interval of consecutive negative labels as $\gamma$ to the top leaf which lies in the same interval of consecutive positive labels as $\gamma$. See Figure 8 . A multiset $\Gamma$ of gaps therefore defines a lamination $\lambda(\mathrm{T}, \Gamma)$ of $\mathrm{T}$, i.e. a multiset of pairwise non-crossing geodesic vertical paths in $\mathrm{T}$ from down leaves to up leaves. When cut along the paths of a lamination, the Cambrian tree $\mathrm{T}$ splits into a forest.

Consider two Cambrian trees T and $\mathrm{T}^{\prime}$ on $[n]$ and $\left[n^{\prime}\right]$ respectively. For any shuffle $s$ of their signatures $\varepsilon$ and $\varepsilon^{\prime}$, consider the multiset $\Gamma$ of gaps of $[n]$ given by the positions of the negative signs of $\varepsilon^{\prime}$ in $s$ and the multiset $\Gamma^{\prime}$ of gaps of $\left[n^{\prime}\right]$ given by the positions of the positive signs of $\varepsilon$ in $s$. We denote by $\mathrm{T} / s \mathrm{~T}^{\prime}$ the Cambrian tree obtained by connecting the up leaves of the forest defined by the lamination $\lambda(T, \Gamma)$ to the down leaves of the forest defined by the lamination $\lambda\left(\mathrm{T}^{\prime}, \Gamma^{\prime}\right)$. An example is given in Figure 7 .
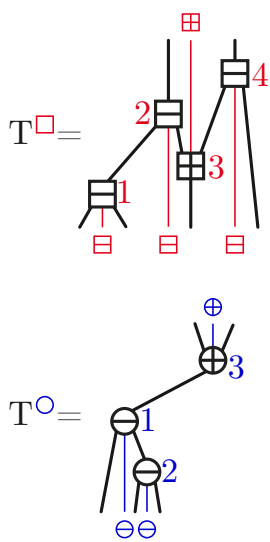

(a)
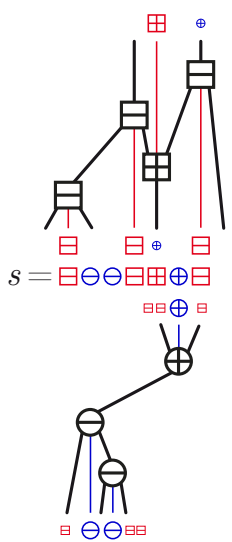

(b)
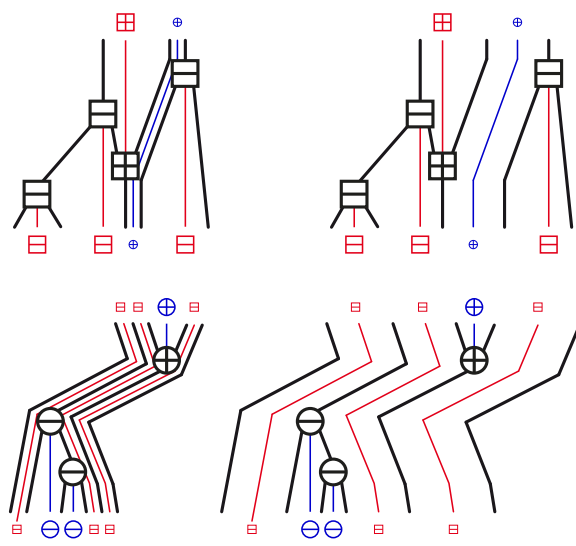

(c)

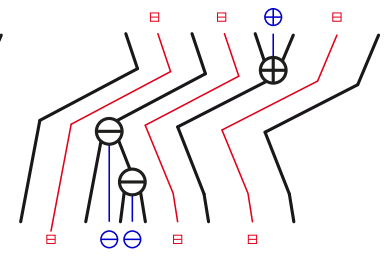

(d)

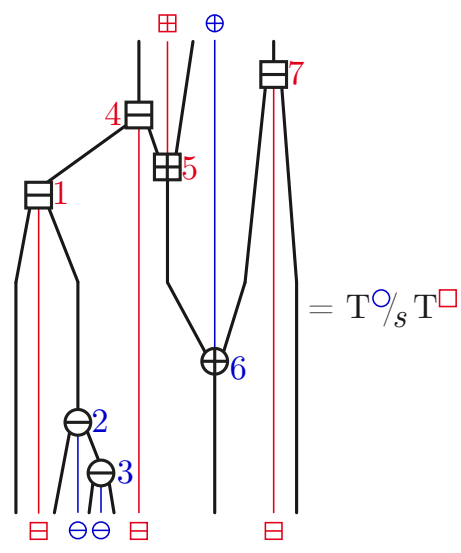

(e)

Fig. 7: (a) Two Cambrian trees $\mathrm{T}^{\bigcirc}$ and $\mathrm{T}^{\square}$. (b) Given the shuffle $s=\boxminus \ominus \ominus \boxminus \boxplus \ominus \boxminus$ of their signatures, the positions of the $\boxminus$ are reported in $\mathrm{T}^{\bigcirc}$ and the positions of the $\oplus$ are reported in $\mathrm{T}^{\square}$. (c) The corresponding laminations. (d) The trees are splitted according to the laminations. (e) The resulting Cambrian tree $T^{\bigcirc} / s T^{\square}$. 
Proposition 19 For any Cambrian trees $\mathrm{T}, \mathrm{T}^{\prime}$, we have $\mathbb{Q}_{\mathrm{T}} \cdot \mathbb{Q}_{\mathrm{T}^{\prime}}=\sum_{s} \mathbb{Q}_{\mathrm{T} / s \mathrm{~T}^{\prime}}$, where s runs over all shuffles of the signatures of $\mathrm{T}$ and $\mathrm{T}^{\prime}$.

For example, we can compute the product

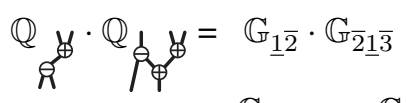

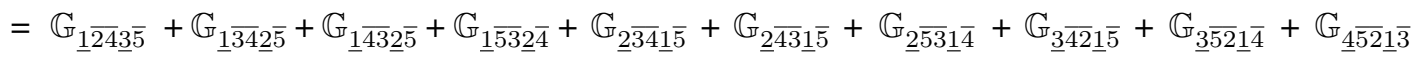

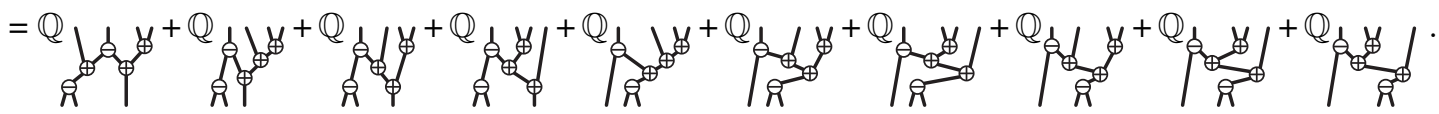

COPRODUCT To describe the coproduct of $\mathbb{Q}$-basis elements of Camb*, we also use gaps and vertical paths in Cambrian trees. Namely, for a gap $\gamma$, we denote by $L(\mathrm{~S}, \gamma)$ and $R(\mathrm{~S}, \gamma)$ the left and right Cambrian subtrees of $\mathrm{S}$ when split along the path $\lambda(\mathrm{S}, \gamma)$. An example is given in Figure 8
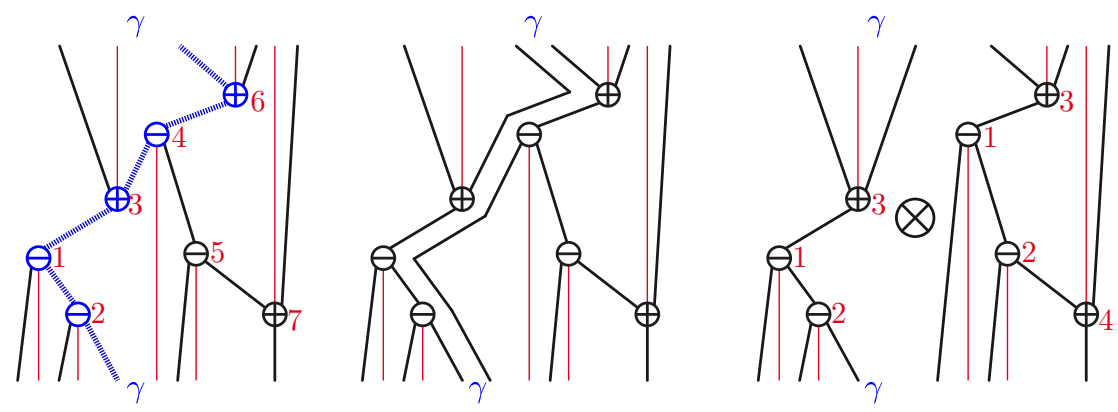

Fig. 8: A gap $\gamma$ between 3 and 4 (left) defines a vertical cut (middle) which splits the Cambrian tree (right).

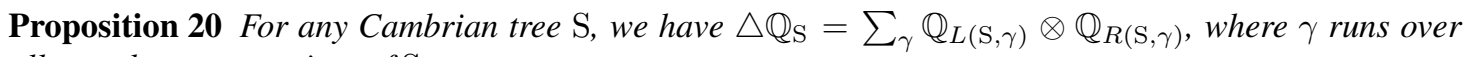
all gaps between vertices of $\mathrm{S}$.

For example, we can compute the coproduct

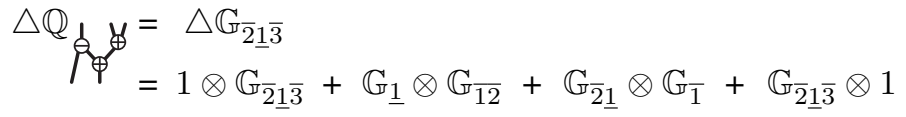

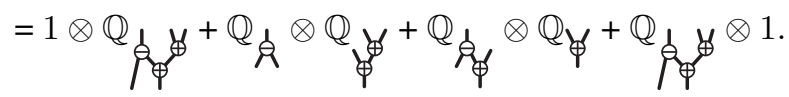

Note that the last line can indeed be directly computed using the paths defined by the four possible gaps of the Cambrian tree Pे $^{\prime}$. 


\subsection{Multiplicative bases}

To conclude, we define multiplicative bases of Camb and study structural and enumerative properties of the indecomposable elements of Camb for these bases. For a Cambrian tree $\mathrm{T}$, we define

$$
\mathbb{E}^{\mathrm{T}}:=\sum_{\mathrm{T} \leq \mathrm{T}^{\prime}} \mathbb{P}_{\mathrm{T}^{\prime}} \quad \text { and } \quad \mathbb{H}^{\mathrm{T}}:=\sum_{\mathrm{T}^{\prime} \leq \mathrm{T}} \mathbb{P}_{\mathrm{T}^{\prime}}
$$

Proposition $21\left(\mathbb{E}^{\mathrm{T}}\right)$ and $\left(\mathbb{H}^{\mathrm{T}}\right)$ are multiplicative bases of Camb: $\mathbb{E}^{\mathrm{T}} \cdot \mathbb{E}^{\mathrm{T}^{\prime}}=\mathbb{E}^{\mathrm{T} \backslash \overline{\mathrm{T}}^{\prime}}$ and $\mathbb{H}^{\mathrm{T}} \cdot \mathbb{H}^{\mathrm{T}^{\prime}}=\mathbb{H}^{\mathrm{T} / \overline{\mathrm{T}}^{\prime}}$.

As the multiplicative bases $\left(\mathbb{E}^{\mathrm{T}}\right)_{\mathrm{T} \in \mathrm{Camb}}$ and $\left(\mathbb{H}^{\mathrm{T}}\right)_{\mathrm{T} \in \mathrm{Camb}}$ have symmetric properties, we focus our analysis on the $\mathbb{E}$-basis. The reader is invited to translate the results below to the $\mathbb{H}$-basis. We consider multiplicative decomposability. Remember that an edge cut in a Cambrian tree $\mathrm{S}$ is the ordered partition $(X \| Y)$ of the vertices of $\mathrm{S}$ into the set $X$ of vertices in the source set and the set $Y$ of vertices in the target set of an oriented edge $e$ of $\mathrm{S}$.

Proposition 22 The following properties are equivalent for a Cambrian tree $\mathrm{S}$ :

(i) $\mathbb{E}^{\mathrm{S}}$ can be decomposed into a product $\mathbb{E}^{\mathrm{S}}=\mathbb{E}^{\mathrm{T}} \cdot \mathbb{E}^{\mathrm{T}^{\prime}}$ for non-empty Cambrian trees $\mathrm{T}, \mathrm{T}^{\prime}$;

(ii) $([k] \|[n] \backslash[k])$ is an edge cut of $\mathrm{S}$ for some $k \in[n]$;

(iii) at least one linear extension $\tau$ of $\mathrm{S}$ is decomposable, i.e. $\tau([k])=[k]$ for some $k \in[n]$.

The tree $\mathrm{S}$ is then called $\mathbb{E}$-decomposable.

For example, Figure 6 shows that $\mathbf{P}(\underline{2} \overline{7} \underline{51} \overline{3} \underline{\underline{6}})$ is $\mathbb{E}$-decomposable. We denote by $\operatorname{Ind}_{\varepsilon}$ the set of $\mathbb{E}$-indecomposable elements of $\operatorname{Camb}(\varepsilon)$.

Example 23 For $\varepsilon=(-)^{n}$, the $\mathbb{E}$-indecomposable $\varepsilon$-Cambrian trees are right-tilting binary trees, i.e. binary trees whose root has no left child. Similarly, for $\varepsilon=(+)^{n}$, the $\mathbb{E}$-indecomposable $\varepsilon$-Cambrian trees are left-tilting binary trees oriented upwards. See Figure 9 for illustrations.

Proposition 24 For any signature $\varepsilon \in \pm^{n}$, the set $\operatorname{Ind}_{\varepsilon}$ of $\mathbb{E}$-indecomposable $\varepsilon$-Cambrian trees forms a principal upper ideal of the $\varepsilon$-Cambrian lattice. See Figure 9
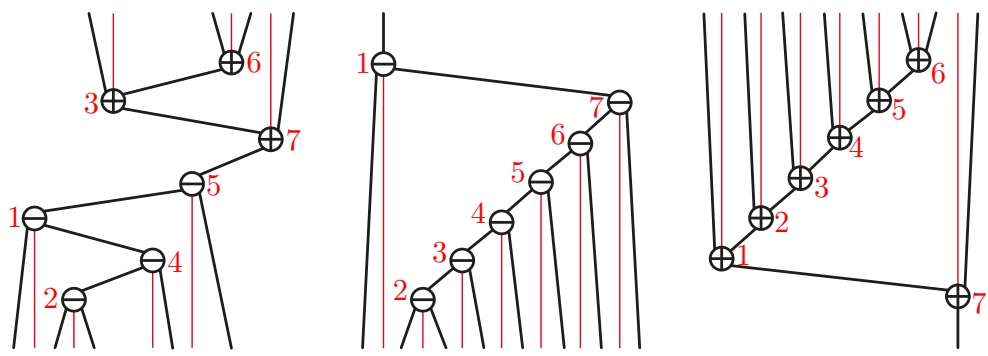

Fig. 9: Generators of the principal upper ideals of $\mathbb{E}$-indecomposable $\varepsilon$-Cambrian trees for $\varepsilon=--+--++$ (left), $\varepsilon=(-)^{7}$ (middle), and $\varepsilon=(+)^{7}$ (right).

Proposition 25 For any signature $\varepsilon \in \pm^{n}$, there are $C_{n-1} \mathbb{E}$-indecomposable $\varepsilon$-Cambrian trees. Therefore, there are $2^{n} C_{n-1} \mathbb{E}$-indecomposable Cambrian trees on $n$ vertices.

Corollary 26 The Cambrian algebra Camb is free. 


\section{Acknowledgements}

We are grateful to the participants of the Groupe de travail de Combinatoire Algébrique de l'Université de

Marne-la-Vallée for helpful discussions on preliminary stages of this work. In particular, we are indebted to J.-Y. Thibon, J.-C. Novelli, and V. Pons for uncountable suggestions, ideas and comments.

\section{References}

[BPR12] François Bergeron and Louis-François Préville-Ratelle. Higher trivariate diagonal harmonics via generalized Tamari posets. J. Comb., 3(3):317-341, 2012.

[CD06] Michael P. Carr and Satyan L. Devadoss. Coxeter complexes and graph-associahedra. Topology Appl., 153(12):2155-2168, 2006.

[CP14] Grégory Chatel and Vincent Pilaud. The Cambrian and Baxter-Cambrian Hopf Algebras. Preprint, arXiv:1411.3704, 2014.

[HL07] Christophe Hohlweg and Carsten Lange. Realizations of the associahedron and cyclohedron. Discrete Comput. Geom., 37(4):517-543, 2007.

[HNT05] Florent Hivert, Jean-Christophe Novelli, and Jean-Yves Thibon. The algebra of binary search trees. Theoret. Comput. Sci., 339(1):129-165, 2005.

[IO13] Kiyoshi Igusa and Jonah Ostroff. Mixed cobinary trees. Preprint, arXiv:1307.3587, 2013.

[Lod04] Jean-Louis Loday. Realization of the Stasheff polytope. Arch. Math. (Basel), 83(3):267-278, 2004.

[LP13] Carsten Lange and Vincent Pilaud. Using spines to revisit a construction of the associahedron. Preprint, arXiv:1307.4391, 2013.

[LR98] Jean-Louis Loday and María O. Ronco. Hopf algebra of the planar binary trees. Adv. Math., 139(2):293-309, 1998.

[MR95] Claudia Malvenuto and Christophe Reutenauer. Duality between quasi-symmetric functions and the Solomon descent algebra. J. Algebra, 177(3):967-982, 1995.

[NT14] Jean-Christophe Novelli and Jean-Yves Thibon. Hopf algebras of $m$-permutations, $(m+1)$-ary trees, and $m$-parking functions. Preprint, arXiv:1403.5962, 2014.

[Pos09] Alexander Postnikov. Permutohedra, associahedra, and beyond. Int. Math. Res. Not. IMRN, (6):1026-1106, 2009.

[Rea05] Nathan Reading. Lattice congruences, fans and Hopf algebras. J. Combin. Theory Ser. A, 110(2):237-273, 2005.

[Rea06] Nathan Reading. Cambrian lattices. Adv. Math., 205(2):313-353, 2006.

[Sol76] Louis Solomon. A Mackey formula in the group ring of a Coxeter group. J. Algebra, 41(2):255264, 1976. 